\title{
Deconstructing the Antlia cluster core ${ }^{\star}$
}

\author{
J. P. Caso ${ }^{1,2}$ and T. Richtler ${ }^{3}$ \\ ${ }^{1}$ Grupo de Investigación CGGE, Facultad de Ciencias Astronómicas y Geofísicas de la Universidad Nacional de La Plata, and \\ Instituto de Astrofísica de La Plata (CCT La Plata - CONICET, UNLP), Paseo del Bosque S/N, B1900FWA La Plata, Argentina \\ 2 Consejo Nacional de Investigaciones Científicas y Técnicas, Rivadavia 1917, C1033AAJ Ciudad Autónoma de Buenos Aires, \\ Argentina \\ 3 Departamento de Astronomía, Universidad de Concepción, Casilla 160-C Concepción, Chile
}

Received 7 August 2015 / Accepted 23 September 2015

\section{ABSTRACT}

Context. The present literature does not give a satisfactory answer to the question about the nature of the "Antlia galaxy cluster". Aims. The radial velocities of galaxies found in the region around the giant ellipticals NGC 3258/3268 range from about $1000 \mathrm{~km} \mathrm{~s}^{-1}$ to $4000 \mathrm{~km} \mathrm{~s}^{-1}$. We characterise this region and its possible kinematical and population substructure.

Methods. We have obtained VLT-VIMOS multi-object spectra of the galaxy population in the inner part of the Antlia cluster and measure radial velocities for 45 potential members. We supplement our galaxy sample with literature data, ending up with 105 galaxy velocities.

Results. We find a large radial velocity dispersion for the entire sample as reported in previous papers. However, we find three groups at about $1900 \mathrm{~km} \mathrm{~s}^{-1}, 2800 \mathrm{~km} \mathrm{~s}^{-1}$, and $3700 \mathrm{~km} \mathrm{~s}^{-1}$, which we interpret as differences in the recession velocities rather than peculiar velocities.

Conclusions. The high radial velocity dispersion of galaxies in the Antlia region reflects a considerable extension along the line of sight.

Key words. galaxies: clusters: individual: Antlia cluster - galaxies: distances and redshifts

\section{Introduction}

The character of the galaxy assembly in the constellation of Antlia is not as clear as, for example, in the cases of the Virgo or Fornax galaxy clusters. Some authors used the term "galaxy group" (Ferguson \& Sandage 1990), others "galaxy cluster" (Hopp \& Materne 1985; Smith Castelli et al. 2008), and Hopp \& Materne (1985) identified five "clusters" in the Antlia region $\left(\alpha=10^{\mathrm{h}}-10^{\mathrm{h}} 50^{\mathrm{m}}, \delta=-42^{\circ}--30^{\circ}\right)$. The most striking central cluster is called "Antlia II". Its morphological appearance is that of two groups concentrated around the giant ellipticals NGC 3258 and NGC 3268 in a projected distance of $220 \mathrm{kpc}$.

Both dominant galaxies are extended X-ray sources (Nakazawa et al. 2000; Pedersen et al. 1997) that exhibit rich globular cluster systems. The globular cluster system of NGC 3258 contains about 6000 members. The system of NGC 3268 is somewhat poorer, but is still typical of a giant elliptical galaxy, with almost 5000 globular clusters (Bassino et al. 2008). Hopp \& Materne (1985) and, more recently, Hess et al. (2015) estimated velocity dispersions of $\sim 500 \mathrm{~km} \mathrm{~s}^{-1}$ for the bright population of Antlia, higher than those in clusters like Fornax (Drinkwater et al. 2001). The globular cluster luminosity function indicated that NGC 3258 could be a few Mpc nearer than NGC 3268 (Dirsch et al. 2003b; Bassino et al. 2008), which agrees with the distances obtained with surface brightness fluctuations by Blakeslee et al. (2001). However, Cantiello et al. (2005) quoted the same distance moduli for both galaxies.

Despite being nearby, a thorough radial velocity survey of Antlia's galaxy population has not been done yet. In this paper,

\footnotetext{
* Appendix $\mathrm{A}$ is available in electronic form at http: //www . aanda.org
}

we present new radial velocities of galaxies located in Antlia. We supplement our galaxy sample with literature data, ending up with 105 galaxy velocities. These data will help us to better understanding the structure of Antlia.

In the following, we keep the term "Antlia cluster" for simplicity. To maintain consistency with earlier papers (e.g. Smith Castelli et al. 2012; Caso et al. 2013, 2014; Calderón et al. 2015), we adopt a distance of $35 \mathrm{Mpc}$, which means a scale of $169.7 \mathrm{pc} / \mathrm{arcsec}$.

\section{Observations and reductions}

We performed multi-object spectroscopy with VLT-VIMOS of the galaxy population in six fields located in the inner part of the Antlia cluster. The observations were carried out under the programmes 60.A-9050(A) and 079.B-0480(B) (PI Tom Richtler), observed during the first semesters of 2007 and 2008. Figure 1 shows the four quadrants for each one of the six VIMOS fields, using different colours.

The grating was HR blue, and the slit width was $1^{\prime \prime}$. For each science field, the integration time was $1 \mathrm{~h}$, split into three individual exposures. This configuration implied a wavelength coverage spanning $3700 \AA-6600 \AA$ (depending on the slit positions) and a spectral resolution of $\sim 2.5 \AA$. The data were reduced with ESOREX in the usual manner for VIMOS data. First, a master bias was obtained for each field with the recipe VMBIAS from five individual bias exposures. The normalised master flat field was created with the recipe VMSPFLAT from a set of dome flat-field exposures. The recipe VMSPCALDISP was used to determine the wavelength calibrations and spectral distortions. Typically, more than 20 lines were identified for each 


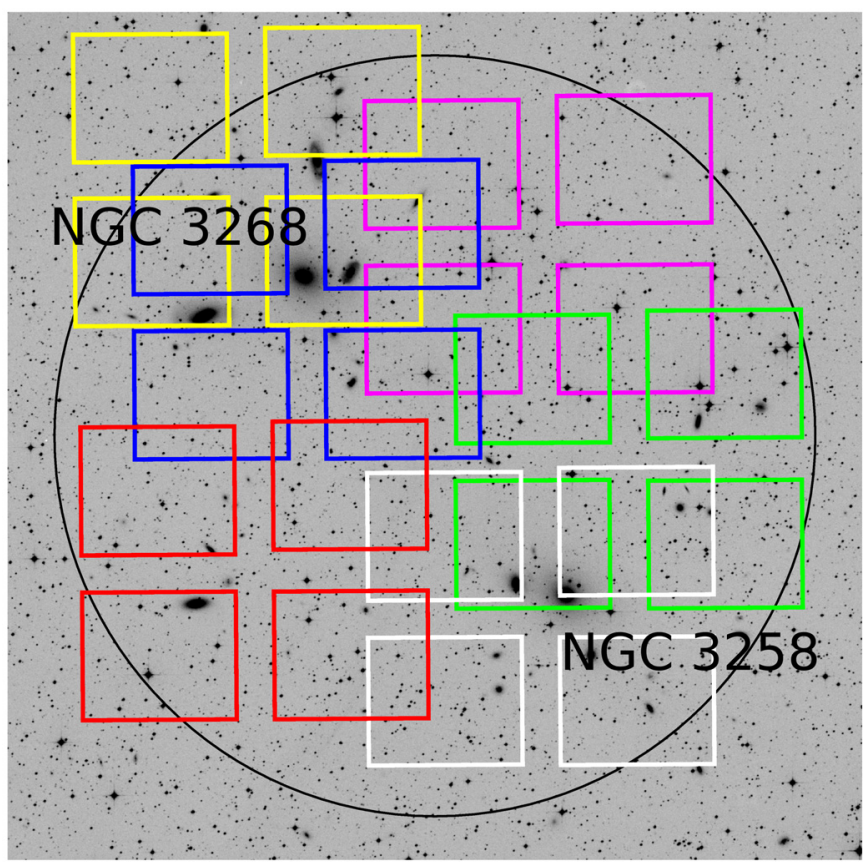

Fig. 1. Positions of the six VIMOS fields (each one composed of four quadrants). The black circle is centred on the midpoint between the projected positions for the two gEs, and its radius is $20^{\prime}$. North is up, east to the left.

slit. Afterwards, the bias and flat-field corrections were applied to each science exposure, together with the wavelength calibration. This was done with the recipe VMMOSOBSSTARE. Individual exposures were then combined with the IRAF task IMCOMBINE to achieve a higher S/N. The spectra were extracted with the task APALL, also within IRAF. We measured the heliocentric radial velocities using the IRAF task FXCOR within the NOAO.RV package. We used synthetic templates, which were selected from the single stellar population (SSP) model spectra at the MILES library ${ }^{1}$ (Sánchez-Blázquez et al. 2006). We selected SSP models with the metallicities $[\mathrm{M} / \mathrm{H}]=-0.71$ and $[\mathrm{M} / \mathrm{H}]=-0.4$, a unimodal initial mass function with slope 1.30 , and an age of $10 \mathrm{Gyr}$. The wavelength coverage of these templates is $3700 \AA-6500 \AA$, and their spectral resolution is $3 \AA$ FWHM.

We also obtained GEMINI-GMOS multi-object spectra from programme GS-2013A-Q-37 (PI J. P. Calderón). The grating B600_G5303 blazed at $5000 \AA$ was used with a slit width of 1 arcsec. The wavelength coverage spans $3300 \AA-7200 \AA$, depending on the position of the slits. The data were reduced using the GEMINI.GMOS package within IRAF. We refer to Caso et al. (2014) for more information about the reduction.

We could determine heliocentric radial velocities $\left(V_{\mathrm{R}, \mathrm{h}}\right)$ for 67 galaxies located in the inner $20^{\prime}$ of the Antlia cluster (i.e., the inner $200 \mathrm{kpc}$ for our adopted distance). In previous studies (Smith Castelli et al. 2008, 2012; Caso et al. 2013), those galaxies with $V_{\mathrm{R}, \mathrm{h}}$ between $1200 \mathrm{~km} \mathrm{~s}^{-1}$ and $4200 \mathrm{~km} \mathrm{~s}^{-1}$ have been assigned to Antlia (which already raised doubts owing to the large velocity interval). In our enhanced sample, we find the lowest velocity to be $V_{\mathrm{R}, \mathrm{h}}=1150 \mathrm{~km} \mathrm{~s}^{-1}$, and there are no velocities between $4300 \mathrm{~km} \mathrm{~s}^{-1}$ and $\sim 7600 \mathrm{~km} \mathrm{~s}^{-1}$. Galaxies with higher $V_{\mathrm{R}, \mathrm{h}}$ than the latter limit were rejected from our sample and are listed in Table A.1. It can be noticed that several galaxies

\footnotetext{
1 http://www.iac.es/proyecto/miles
}

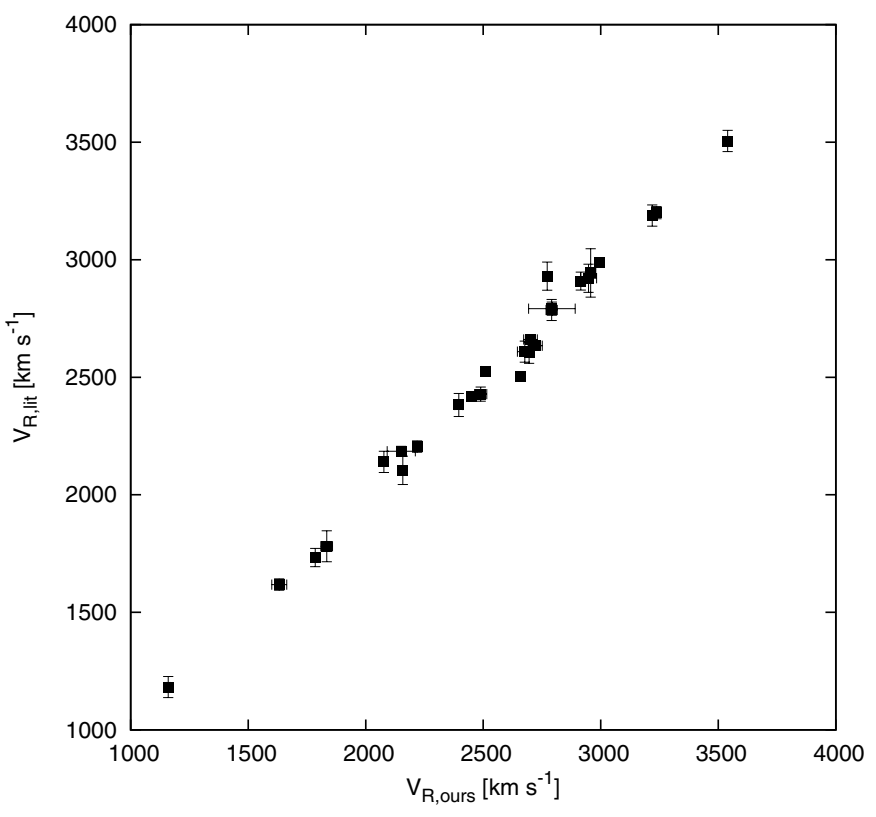

Fig. 2. Comparison between heliocentric radial velocity measurements from this paper and the literature for Antlia members.

from the Ferguson \& Sandage (1990) catalogue are indeed in the background. In these cases, Ferguson \& Sandage (1990) classified them as "likely members" or "probable background". From the 67 galaxies with $V_{\mathrm{R}, \mathrm{h}}$ measurements, 45 are thus members of our sample.

Additional $V_{\mathrm{R}, \mathrm{h}}$ measurements were collected from the literature (Smith Castelli et al. 2008, 2012) and the NED ${ }^{2}$. The final sample of Antlia members consists of 105 galaxies, which are listed in Table A.2. A fraction of the present galaxies had been measured earlier. In these cases, we find good agreement between our measurements and the literature values (Fig. 2). The mean $V_{\mathrm{R}, \mathrm{h}}$ difference and dispersion are $20 \mathrm{~km} \mathrm{~s}^{-1}$ and $50 \mathrm{~km} \mathrm{~s}^{-1}$, respectively. In comparison, the uncertainties of our measurements are typically in the range $10-40 \mathrm{~km} \mathrm{~s}^{-1}$.

Ferguson \& Sandage (1990) provided the photometrical catalogue of galaxies in Antlia with the largest spatial coverage, while Calderón et al. (2015) have carried out a deeper survey of the early-type galaxies in a region that contains our VIMOS fields. To supplement the Ferguson \& Sandage (1990) catalogue, we derived $B$ magnitudes for the galaxies measured by Calderón et al. (2015) in the Washington $\left(C, T_{1}\right)$ photometric system (Canterna 1976). As a result, we applied equation 4 from Smith Castelli et al. (2008) to transform $\left(C-T_{1}\right)_{0}$ into $(B-R)_{0}$ colours. Then we obtained $B$ magnitudes, considering that $R$ and $T_{1}$ filters only differ in a small offset (Dirsch et al. 2003b). Figure 3 shows the completeness for those galaxies located within $30^{\prime}$ of the cluster centre (see Sect. 3.2), which roughly matches the region observed with our VIMOS fields and previous spectroscopic studies (Smith Castelli et al. 2008, 2012). The bin width is $1 \mathrm{mag}$. When we exclude galaxies with Ferguson \& Sandage (1990) membership status "3" (which are the less probable members), the $80 \%$ completeness is reached at $B_{T}=17 \mathrm{mag}$ and the $60 \%$ at $B_{T}=19 \mathrm{mag}$ (small red circles).

2 This research has made use of the NASA/IPAC Extragalactic Database (NED), which is operated by the Jet Propulsion Laboratory, California Institute of Technology, under contract with the National Aeronautics and Space Administration. 


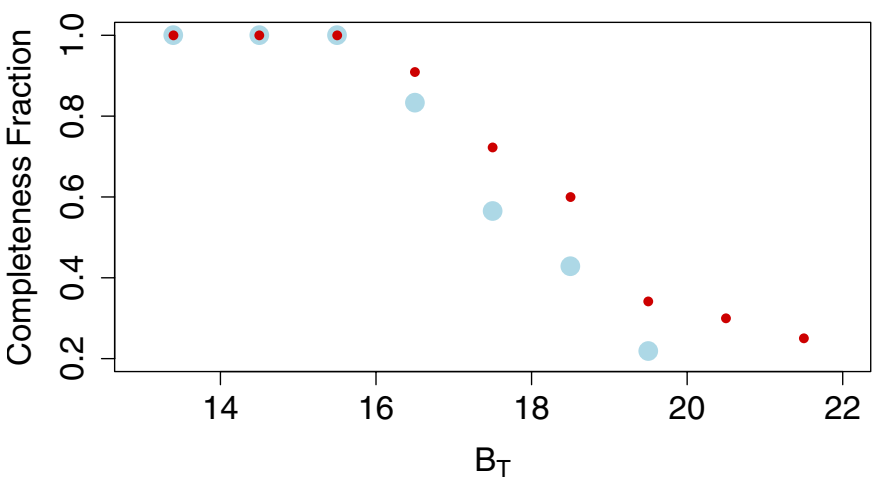

Fig. 3. Completeness analysis for the spectroscopic sample considering the entire photometric catalogue (large light-blue circles), and excluding galaxies with Ferguson \& Sandage (1990) membership status "3" (small red circles). The bin width is 1 mag.

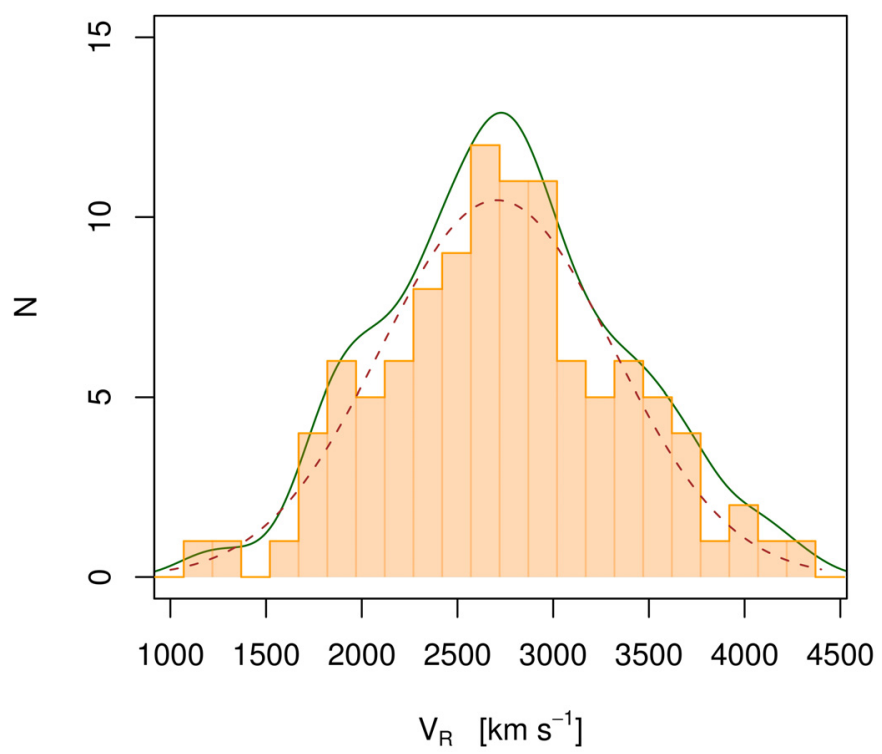

Fig. 4. Histogram of the $V_{\mathrm{R}, \mathrm{h}}$ distribution for the galaxies in our Antlia sample. The bin width is $150 \mathrm{~km} \mathrm{~s}^{-1}$. Overplotted are the smooth velocity distribution, obtained with a Gaussian kernel (green solid curve), and the normal profile fitted by least squares (brown dashed curve).

\section{Results}

\section{1. $V_{R, h}$ velocity distribution}

The histogram of the $V_{\mathrm{R}, \mathrm{h}}$ distribution for the galaxies in our Antlia sample is shown in Fig. 4 with a bin width of $150 \mathrm{~km} \mathrm{~s}^{-1}$. The green solid curve represents the smooth velocity distribution, obtained with a Gaussian kernel. The distribution resembles a Gaussian, which would be expected if the spatial velocity for the Antlia members is described by a Maxwellian distribution.

A Shapiro-Wilk normality test returns a $p$ value of 0.9 (Shapiro \& Wilk 1965; Royston 1995, hereafter S-W test), meaning that we cannot reject the hypothesis that our sample is drawn from a normal distribution. Considering this, we fitted a normal profile to the $V_{\mathrm{R}, \mathrm{h}}$ distribution by least-squares, assuming Poisson uncertainties for the bin values. The best fit corresponds to a Gaussian distribution with a mean velocity of $2708 \pm 42 \mathrm{~km} \mathrm{~s}^{-1}$ and a dispersion of $617 \pm 46 \mathrm{~km} \mathrm{~s}^{-1}$ (dashed brown curve in Fig. 4), which agree with the values from optical data by Hess et al. (2015). This dispersion fits massive clusters like Virgo (Conselice et al. 2001). Nakazawa et al. (2000) obtained a gas temperature of $2 \mathrm{keV}$ for the surroundings of NGC 3268, which does not exclude a dispersion of $617 \mathrm{~km} \mathrm{~s}^{-1}$, considering the $\sigma$-T-relation of Xue \& Wu (2000) and its scatter. However, whether this high velocity dispersion obtained for our Antlia sample represents a single virialized system, is an interesting question, which we try to answer here.

Assuming that the individual $V_{\mathrm{R}, \mathrm{h}}$ uncertainties are the sigma values of a normal distribution, we simulated the $V_{\mathrm{R}, \mathrm{h}}$ for each one of the 105 galaxies by a Monte-Carlo method. The procedure was repeated 100 times, in all cases obtaining a nonrejection of the normality hypothesis. This demonstrates that the intrinsic uncertainties of the measurements do not play a central role in the result.

Several studies in galaxy clusters found different velocity dispersions for the dwarf and bright galaxies populations (e.g. Drinkwater et al. 2001; Conselice et al. 2001; Edwards et al. 2002). To test whether this is also true for the Antlia cluster, we subdivided our sample in giants and dwarfs using the morphological classification from Smith Castelli et al. (2008, 2012) and, when this is not available, from Ferguson \& Sandage (1990). Normal distributions were fitted by least squares to the dwarf and bright galaxies, separately. The resulting mean values for the bright and dwarf populations were $2768 \pm 104 \mathrm{~km} \mathrm{~s}^{-1}$ and $2698 \pm 108 \mathrm{~km} \mathrm{~s}^{-1}$, respectively. The corresponding dispersions are $698 \pm 122 \mathrm{~km} \mathrm{~s}^{-1}$ and $682 \pm 120 \mathrm{~km} \mathrm{~s}^{-1}$. From a K-S test, we cannot reject the hypothesis that both samples are drawn from the same distribution with $90 \%$ confidence.

\subsection{Morphological types and spatial distribution}

Assuming the groups of galaxies dominated by the gEs as the main substructures of the cluster and that both haloes present similar masses (Pedersen et al. 1997; Nakazawa et al. 2000), we consider the midpoint between them as its approximate centre $\left(\alpha=10^{\mathrm{h}} 29^{\mathrm{m}} 27^{\mathrm{s}}, \delta=-35^{\circ} 27^{\prime} 58^{\prime \prime}\right)$.

The 105 galaxies in our sample occupy a region of $\sim 7 \mathrm{deg}^{2}$. Therefore, we obtained the mean surface density, $\sim 15 \mathrm{deg}^{-2}$, and the mean distance between galaxies, $\sim 9$ arcmin. For each galaxy, we calculated the number of neighbours nearer than this value, and we used it to split the galaxies in four percentiles. The left-hand panel of Fig. 5 shows the regions occupied by the 25,50 , and 75 percentiles of galaxies with a larger number of neighbours. The white asteriks correspond to the position of NGC 3258 (south-west) and NGC 3268 (north-east). The origin of the black circle is the bona fide centre of the cluster, and its radius is $30^{\prime}$ (i.e. $\sim 300 \mathrm{kpc}$ at Antlia distance). The 25 percentile seems to clearly represent the overdensities of galaxies around both gEs, which are also identified in the 50th percentile. The lower percentile shows a more extended spatial distribution.

In the middle panel of Fig. 5, the projected spatial distribution for bright galaxies (i.e., all those galaxies not classified as dwarfs) is plotted. Different symbols indicate the Hubble type (i.e., spirals, lenticulars, or ellipticals). The colour palette ranges from red to blue, spanning the $V_{\mathrm{R}, \mathrm{h}}$ range defined in Sect. 2 . The origin and radius of the black circle are identical to those in the left-hand panel. The two groups around each of the gEs can be clearly identified. There are also several galaxies located in the extrapolation of the straight line joining the two gEs, mainly to the north-east. These galaxies, as well as those located in the central part of the cluster, mainly present intermediate $V_{\mathrm{R}, \mathrm{h}}$. There are a few galaxies with $V_{\mathrm{R}, \mathrm{h}}<2000\left[\mathrm{~km} \mathrm{~s}^{-1}\right.$, whose projected positions agree with the general scheme of galaxies with intermediate velocities. However, the galaxies with the higher $V_{\mathrm{R}, \mathrm{h}}$ seem to present a different projected spatial distribution. 

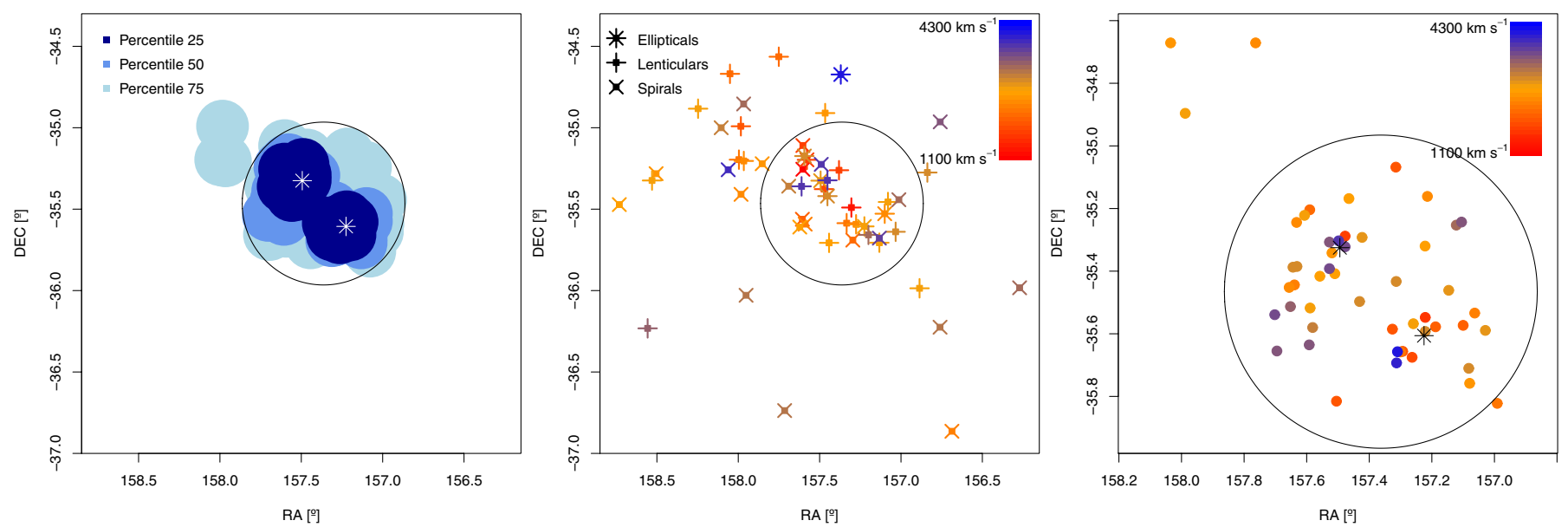

Fig. 5. Left panel: projected density distribution for galaxies, split into three percentile ranges. Middle panel: projected positions for bright galaxies. The colour palette ranges from red to blue, spanning the $V_{\mathrm{R}, \mathrm{h}}$ range defined in Sect. 2. Right panel: similar plot for dwarf galaxies in the sample. The black asterisks indicate the positions of NGC 3258 (south-west) and NGC 3268 (north-east). In both panels, the origin of the black circle is the bona fide centre of the cluster, and its radius is $30^{\prime}$.

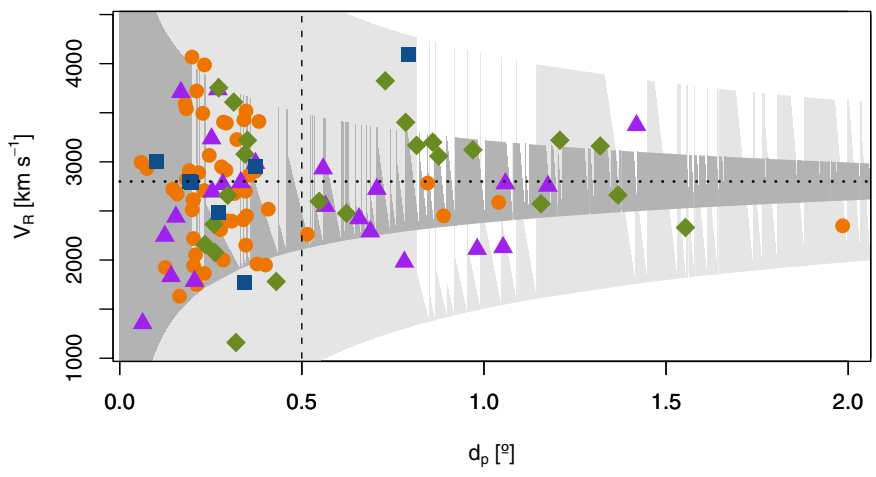

Fig. 6. Heliocentric radial velocities $\left(V_{\mathrm{R}, \mathrm{h}}\right)$ as a function of projected distances to the galaxy centre $\left(d_{\mathrm{p}}\right)$. Different symbols represent dwarf (red circles), ellipticals (blue squares), lenticulars (blue-violet triangles), and spirals (green diamonds). The dashed line indicates $d_{\mathrm{p}}=30^{\prime}$, and the dotted one represents the $V_{\mathrm{R}, \mathrm{h}}$ for both $\mathrm{gEs}, \sim 2800 \mathrm{~km} \mathrm{~s}^{-1}$. The grey regions indicate the caustic curves obtained with the Praton \& Schneider (1994) infall model for two sets of parameters.

The majority of them were classified as spirals, despite the relatively isolated elliptical FS90-152 (see Table A.2). On the other hand, the few other ellipticals have a projected distance to the centre of the cluster $\left(d_{\mathrm{p}}\right)$ lower than $25^{\prime}$ and present $1775<$ $V_{\mathrm{R}, \mathrm{h}}\left[\mathrm{km} \mathrm{s}^{-1}\right]<3000$.

The right-hand panel of Fig. 5 shows the projected spatial distribution for dwarf galaxies in the sample and the positions of NGC 3258 and NGC 3268. The origin and radius of the black circle are identical to the previous panels. Their concentration within the region restricted by $d_{\mathrm{p}}=30^{\prime}$ is due to a bias in the spectroscopic survey. Therefore, we cannot arrive at any conclusion regarding their distribution when $d_{\mathrm{p}}>30^{\prime}$. The dwarfs near the centre present a wide range of $V_{\mathrm{R}, \mathrm{h}}$. They seem to be more concentrated towards the two gEs and do not seem to be aligned with the projected position of the two gEs.

Figure 6 shows the $V_{\mathrm{R}, \mathrm{h}}$ as a function of $d_{\mathrm{p}}$ for the galaxies in the sample, discriminated by morphological types. The grey regions represent caustic curves obtained with the infall model of Praton \& Schneider (1994), assuming $\Omega_{0}=0.3$ and two sets of parameters. For the inner region, we proposed typical parameters for the Fornax cluster $r_{\mathrm{vir}}=0.7 \mathrm{Mpc}$ and $\sigma_{v}=374 \mathrm{~km} \mathrm{~s}^{-1}$
(Drinkwater et al. 2001). Considering the high mass derived for the Antlia cluster by Hess et al. (2015), we plotted the second region assuming the Virgo cluster virial radius, $r_{\mathrm{vir}}=1.8 \mathrm{Mpc}$ (Kim et al. 2014) and $\sigma_{v}=617 \mathrm{~km} \mathrm{~s}^{-1}$, which were previously derived in Sect. 3.1. While the first set of parameters implies that a large number of galaxies lie outside of the caustic curves, for the second one all galaxies present $V_{\mathrm{R}, \mathrm{h}}$-values lower than the escape velocity at their corresponding projected distances from the assumed centre of the cluster.

Dwarf galaxies seem to be spread all over the $V_{\mathrm{R}, \mathrm{h}}$ range for $d_{\mathrm{p}}<20^{\prime}$. Just a few dwarf galaxies outside this limit were measured, but all of them present intermediate values of $V_{\mathrm{R}, \mathrm{h}}$. The picture is quite different when we look at the bright galaxy population. As expected, spiral galaxies are mainly at larger distances than $20^{\prime}$ from the bona fide cluster centre. Moreover, their velocity distribution also distinguishes them from the rest of the galaxies in the sample. From the thirteen spirals with $d_{\mathrm{p}}>30^{\prime}$, seven of them present $V_{\mathrm{R}, \mathrm{h}}$ around $3200 \mathrm{~km} \mathrm{~s}^{-1}$ and relatively low dispersion. In fact, the mean $V_{\mathrm{R}, \mathrm{h}}$ and its dispersion for these spirals are $3190 \pm 40 \mathrm{~km} \mathrm{~s}^{-1}$ and $110 \mathrm{~km} \mathrm{~s}^{-1}$.

If we consider all the spirals with $d_{\mathrm{p}}>30^{\prime}$, their mean $V_{\mathrm{R}, \mathrm{h}}$ is $2980 \pm 120 \mathrm{~km} \mathrm{~s}^{-1}$. For comparison, the eleven lenticulars in the same radial regime have a mean velocity of $2550 \pm 125 \mathrm{~km} \mathrm{~s}^{-1}$. A Student's test between the two samples rejects the hypothesis that both samples belong to the same population with $90 \%$ of confidence.

To study the central part of the cluster, which is expected to be dominated by the gEs haloes, we restricted our sample to include the 50th percentile of galaxies with larger number of neighbours. The upper panel of Fig. 7 shows the $V_{\mathrm{R}, \mathrm{h}}$ distribution for these galaxies, adopting a bin width of $180 \mathrm{~km} \mathrm{~s}^{-1}$. It seems to represent three groups of galaxies with $V_{\mathrm{R}, \mathrm{h}}$ around $\sim 2000 \mathrm{~km} \mathrm{~s}^{-1}, \sim 2800 \mathrm{~km} \mathrm{~s}^{-1}$, and $\sim 3700 \mathrm{~km} \mathrm{~s}^{-1}$. To the last group belong the three bright lenticulars NGC 3267 (FS90168), NGC 3269 (FS90-184), and NGC 3271 (FS90-224), near to NGC 3268 in projected distance. Considering the $V_{\mathrm{R}, \mathrm{h}}$ for both gEs, the galaxies in the group with intermediate velocities have the higher probability of belonging to the Antlia cluster. We applied a S-W test to this sample and obtained a $p$ value of 0.32. Then, we ran GMM (Muratov \& Gnedin 2010) for a trimodal case. GMM calculated three peaks with means of $2020 \pm 160 \mathrm{~km} \mathrm{~s}^{-1}, 2750 \pm 100 \mathrm{~km} \mathrm{~s}^{-1}$, and $3650 \pm 50 \mathrm{~km} \mathrm{~s}^{-1}$. 

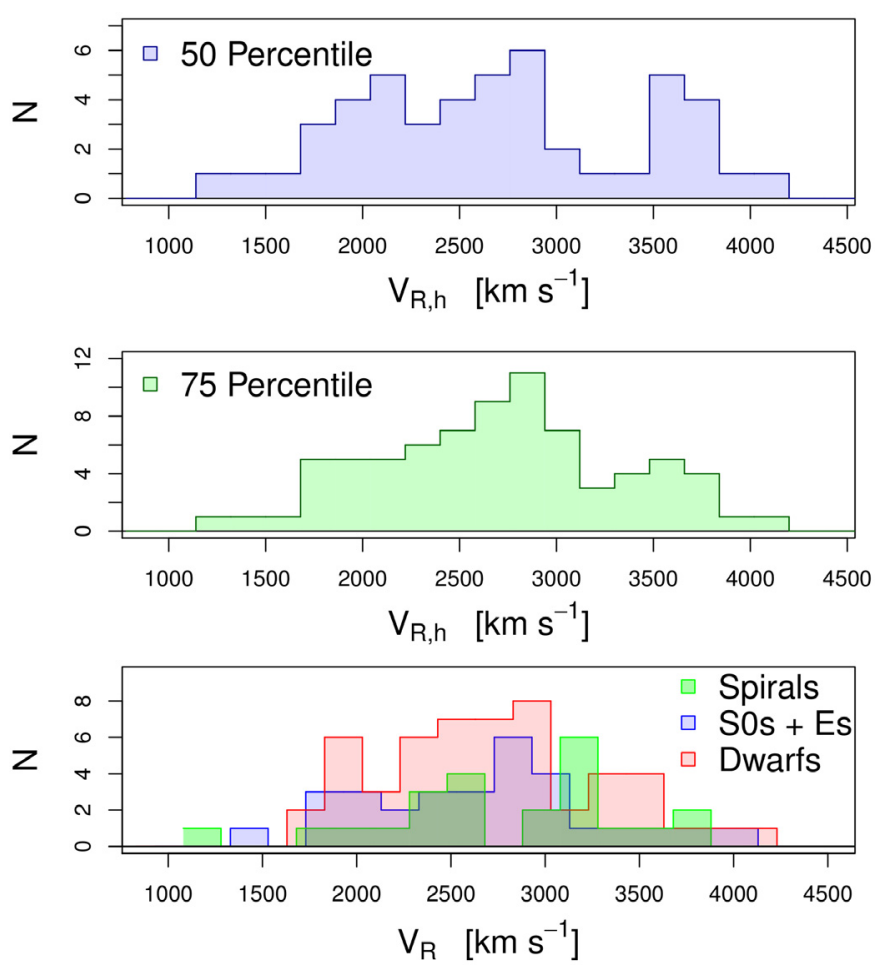

Fig. 7. Upper panel: $V_{\mathrm{R}, \mathrm{h}}$ distribution for galaxies belonging to the 50 percentile with larger number of neighbours. Middle panel: $V_{\mathrm{R}, \mathrm{h}}$ distribution for galaxies belonging to the 75 percentile with larger number of neighbours. Lower panel: $V_{\mathrm{R}, \mathrm{h}}$ distribution for Antlia galaxies, separated by morphology.

For the unimodal hypothesis, we obtained $\chi^{2}=13.9$ with eight degrees of freedom $\left(p \chi^{2}=0.1\right)$ and a distribution kurtosis $k=-0.9$. These results point to a multi-modal distribution. The parameter $D D$, which measures the relevance of the peak detections, was $2.8 \pm 0.7(D D>2$ is required for a meaningful detection; Ashman et al. 1994; Muratov \& Gnedin 2010).

In the middle panel we present the $V_{R, h}$ distribution for galaxies up to the 75 th percentile with the same bin width as in the previous plot. The three different groups also seem to be present, but smoothed. In this case, the $\mathrm{p}$ value obtained from the $\mathrm{S}-\mathrm{W}$ test is higher, $\sim 0.80$. For the trimodal case, the mean from GMM were $2060 \pm 200 \mathrm{~km} \mathrm{~s}^{-1}, 2780 \pm 100 \mathrm{~km} \mathrm{~s}^{-1}$ and $3600 \pm 130 \mathrm{~km} \mathrm{~s}^{-1}$. In this case, $\chi^{2}=7\left(p \chi^{2}=0.5\right), k=-0.5$, and $D D=2.5 \pm 0.8$. The parameters are less conclusive, but point to a multi-modal distribution in $V_{\mathrm{R}, \mathrm{h}}$.

The lower panel of Fig. 7 shows the $V_{R, h}$ distribution for Antlia galaxies, separated between dwarf and bright galaxies, and the latter group between early-types (ellipticals and lenticulars) and late-types (spirals). Spirals fill a wide range of $V_{\mathrm{R}, \mathrm{h}}$, but seem to avoid velocities similar to those of the gEs $\left(\sim 2800 \mathrm{~km} \mathrm{~s}^{-1}\right)$.

\subsection{Mass estimations}

Pedersen et al. (1997) used X-ray observations (ASCA) to measure the mass enclosed within a radius of $240 \mathrm{kpc}$ from NGC 3258 and obtained $0.9-2.4 \times 10^{13} M_{\odot}$. NGC 3268 was studied in X-rays (ASCA) by Nakazawa et al. (2000), who determined $\sim 2 \times 10^{13} M_{\odot}$ internal to $\sim 260 \mathrm{kpc}$. Here we compare our galaxy velocities with the results from X-ray observations. We excluded spirals because of the differences that we found between their $V_{\mathrm{R}, \mathrm{h}}$ distribution and those of early-type bright galaxies in Sect.3.2. Two subsets of our sample were made, selecting from the galaxies contained in the 50th percentile those with shorter projected distance to NGC 3258 or NGC 3268. These results in samples of 17 (up to 10 arcmin) and 24 members (up to 18 arcmin), respectively.

We applied the "tracer mass estimator" $\left(M_{\mathrm{Tr}}\right)$ from Evans et al. (2003),

$M=\frac{C}{G N} \sum_{i} V_{\mathrm{LOS}, i}^{2} R_{i}$,

where $R_{i}$ and $V_{\mathrm{LOS}, i}$ are the projected distances from the corresponding $\mathrm{gE}$ and velocities relative to it, respectively. Here, $G$ is the constant of gravitation, and $N$ the number of tracers. In the case of isotropy, the constant $C$ is calculated through

$C=\frac{4(\alpha+\gamma)}{\pi} \frac{4-\alpha-\gamma}{3-\gamma} \frac{1-\left(r_{\text {in }} / r_{\text {out }}\right)^{3-\gamma}}{1-\left(r_{\text {in }} / r_{\text {out }}\right)^{4-\alpha-\gamma}}$.

We assume that the three-dimensional profile of the tracer population is represented well by a power law between an inner radius $r_{\text {in }}$ and outer radius $r_{\text {out }}$ with exponents $\gamma$ and $\alpha$, respectively (e.g. see Mamon \& Łokas 2005a,b). Under the assumption of constant circular velocity, $\alpha=0$. In the case of the tracer population, it is difficult to measure the power law exponent precisely, but it should not differ too much from $\gamma=3$ (Dekel et al. 2005; Agnello et al. 2014; Courteau et al. 2014). Taking this into account, we obtain the mass that corresponds to $\gamma$ ranging from 2.75 to 3.25 . In the case of a shallower profile, the resulting masses are somewhat lower, but not by an order of magnitude.

For the sample surrounding NGC 3258 we obtained $M_{\operatorname{Tr}}=$ $(8-11) \times 10^{13} M_{\odot}$. This has to be compared with the X-ray mass of NGC 3258. Pedersen et al. (1997) obtained $k T_{\mathrm{e}} \approx 1.7 \mathrm{keV}$, assuming an isothermal gas. The parameters of their beta model are $r_{\mathrm{c}}=8.2$ arcmin, corresponding to $83.5 \mathrm{kpc}$, and $\beta=0.6$.

With the assumption of spherical symmetry, we apply the expression (Grego et al. 2001)

$M(r)=\frac{3 \beta k T_{\mathrm{e}}}{G \mu m_{\mathrm{p}}} \frac{r^{3}}{r_{\mathrm{c}}^{2}+r^{2}}$.

Adopting $\mu=0.6$, the derived mass within the sphere with radius $10^{\prime}$ is $\sim 7 \times 10^{12} M_{\odot}$. In the case of the sample surrounding NGC 3268, the result from the Evans et al. (2003) estimator is $M_{\mathrm{Tr}}=(5-8) \times 10^{13} M_{\odot}$.

We again applied Eq. (3) with the parameters derived by Nakazawa et al. (2000): $k T_{\mathrm{e}}=2 \mathrm{keV}, r_{\mathrm{c}}=5 \mathrm{arcmin}$, and $\beta=0.38$. Then the mass enclosed within $18^{\prime}$ around NGC 3268 is $\sim 1.4 \times 10^{13} M_{\odot}$. In both cases, the mass derived from X-ray observations is significantly lower than the mass estimated from the $V_{\mathrm{R}, \mathrm{h}}$ measurements. It is unlikely that the mass enclosed within $\sim 100 \mathrm{kpc}$ around both gEs reaches values of a few times $10^{13} M_{\odot}$. We therefore conclude that the galaxies with the most deviant velocities in the two samples are probably not gravitationally bound to either of the two groups.

From the $V_{\mathrm{R}, \mathrm{h}}$ histograms in Fig. 7, a large number of the galaxies is symmetrically grouped around 2750 $2800\left[\mathrm{~km} \mathrm{~s}^{-1}\right]$, spanning approximately the velocity range $2200<V_{\mathrm{R}, \mathrm{h}}\left[\mathrm{km} \mathrm{s}^{-1}\right]<3400$. This group corresponds to $\sim 50 \%$ of the galaxies that belong to the 50th percentile, and $\sim 60 \%$ of the galaxies for the 75 th percentile. This velocity constraint reduces the size of the samples around NGC 3258 and NGC 3268 to 8 and 13 members, respectively. Applying the mass estimators to these limited samples, we obtain, for the galaxies, around 
NGC 3258 and NGC $3268 M_{\operatorname{Tr}}=(1.4-2) \times 10^{13} M_{\odot}$ up to $9^{\prime}$ and $M_{\mathrm{Tr}}=(1.7-2.4) \times 10^{13} M_{\odot}$ up to $15^{\prime}$, respectively.

These estimations are in reasonable agreement with the masses derived from X-ray observations. The mass that we derived from the analysis of an UCD sample around NGC 3268 is $2.7 \times 10^{12} M_{\odot}$ within $47 \mathrm{kpc}$. Assuming a constant circular velocity, the extrapolated mass within $101 \mathrm{kpc}$ (corresponding to $10^{\prime}$ ) is $6 \times 10^{12} M_{\odot}$, and therefore also is in reasonable agreement with our mass estimation from both companion galaxies and X-rays. To avoid the need to explain peculiar radial velocities of the order of $1500 \mathrm{~km} \mathrm{~s}^{-1}$, we conclude that the large velocity dispersion of our complete sample is caused by recessional velocities of galaxies in the near foreground or background, which are mixed in.

\subsection{Tests for cluster substructure}

In this section, we try a different approach. The nearestneighbour tests are commonly used for detecting subgroups in the environment of clusters of galaxies (e.g. Boschin et al. 2010, 2006; Burgett et al. 2004; Owers et al. 2009; Hou et al. 2012). Our aim is to search for substructure in $V_{\mathrm{R}, \mathrm{h}}$ besides the obvious existence of the two groups dominated by NGC 3258 and NGC 3268.

The $\Delta$ test (Dressler \& Shectman 1988) was intended for probing deviations from the local mean velocities and dispersions compared with the global cluster values. To achieve this, the local mean velocity and dispersion are calculated for each galaxy, restricting the sample to the galaxy itself and its ten nearest neighbours. Then, their deviation from the global cluster values are computed, and their sum is defined as the $\Delta$ parameter. Colless \& Dunn (1996) proposed the $\kappa$ test, similar in intent to the $\Delta$ test. The possibility that the scale in which the substructure is more obvious differs from what is imposed by the ten nearest neighbours condition is considered, leaving the number of neighbours $n$ as a free parameter. Arguing that distributions cannot be characterised by the first two moments in general, they proposed a statistic ruled by the probability of the KS two-sample distribution (Kolmogorov 1933; Smirnov 1948). For both tests, the significance of their statistics are estimated by Monte Carlo simulations, in which the velocities of the cluster galaxies are shuffled randomly. The significance level $p$ was obtained after repeating the previous procedure 1000 times.

Pinkney et al. (1996) found that the existence of radial gradients in the velocity dispersion of galaxies could produce artificial substructure when applying 3D statistics. We therefore ran the tests on galaxies whose projected distances to the equidistant point to both gEs was less than $20^{\prime}$, because velocity dispersions seem to remain constant up to that limit (see Fig. 6). This restriction reduced our sample to 57 members. The area matches the field of view of our VIMOS survey, which guarantees a homogeneous sample. For the $\kappa$ test, we selected $n=10,15$. The results were $\Delta=66.9$ and $p_{\Delta}=0.12, \kappa_{10}=14.4$ and $p_{10}=0.16$, $\kappa_{15}=15.6$ and $p_{15}=0.06$.

To investigate the significance of these numbers, we performed 1000 Monte Carlo simulations. We randomly generated the $V_{\mathrm{R}, \mathrm{h}}$ for the galaxies in the sample, assuming a normal distribution with mean and dispersion of $2800 \mathrm{~km} \mathrm{~s}^{-1}$ and $275 \mathrm{~km} \mathrm{~s}^{-1}$, respectively. Then, we applied the tests to these samples. For the $\Delta$ test, fewer than $15 \%$ of the cases produced a $\Delta$-parameter higher than the value obtained from the observations, and this percentage is reduced to $6 \%$ for the $\kappa_{15}$ test. Therefore, the tests point to underlying substructure, most probably due to the existence of non-member galaxies in our sample.

\section{Discussion}

\subsection{Extreme radial velocities}

To use radial velocities as a discriminant for cluster membership is often difficult if the depth along the line of sight is considerable. An extreme example is the interesting pair of Virgo galaxies IC 3492 with a radial velocity of $-575 \mathrm{~km} \mathrm{~s}^{-1}$ and IC 3486 with $1903 \mathrm{~km} \mathrm{~s}^{-1}$, having a separation of only $1.4 \mathrm{arc} \min$. The deviation from a strict Hubble law may be a mix of peculiar velocities resulting from large scale structure and local gravitational fields. Is it possible that the extreme radial velocities in Fig. 6 are infall velocities? To answer this, it is useful to consider the highest possible velocities. We can represent the total mass distribution around NGC 3268 quite well by an NFW-mass distribution with a scale length of $22 \mathrm{kpc}$ and a characteristic density of $0.05 M_{\odot} / \mathrm{pc}^{3}$. A mass probe on an exactly radial orbit, initially at rest and falling in from a distance of $1.5 \mathrm{Mpc}$, crosses the centre after $6.8 \mathrm{Gyr}$ with a velocity of $1550 \mathrm{~km} \mathrm{~s}^{-1}$. However, after an additional $0.06 \mathrm{Gyr}$, its velocity has already declined to $1000 \mathrm{~km} \mathrm{~s}^{-1}$. It is obvious that such a configuration is highly artificial and not suitable to explaining the broad velocity interval. The more plausible explanation is therefore the mix of recessional and Doppler velocities.

\subsection{Internal structure of the two dominant groups}

What we called the Antlia cluster seems to be mainly formed by two subgroups, each one dominated by a gE. Despite that, both galaxies present similar $V_{\mathrm{R}, \mathrm{h}}$, it is uncertain whether they are located at the same distance (e.g. Blakeslee et al. 2001; Cantiello et al. 2005; Bassino et al. 2008). A recent study in HI (Hess et al. 2015) indicates that the subgroup dominated by NGC 3258 could be falling in to NGC 3268, which they estimated is the actual cluster centre. It is clear that both gEs dominate their own subgroups, and their peculiar velocities relative to the group systemic velocity should not differ significantly. For this reason, if the difference in distance between both galaxies was $2-4 \mathrm{Mpc}$, we would expect a $V_{R, h}$ difference of $\sim 140-280 \mathrm{~km} \mathrm{~s}^{-1}$. As a result, the measured $V_{\mathrm{R}, \mathrm{h}}$ might agree with the scenario suggested by Hess et al. (2015), where both subgroups are in a merging process.

It is interesting to have a closer look at the group around NGC 3268. Strikingly, all three S0's around the central galaxy (FS90-224, FS90-168, and FS90-184) have positive velocity offsets in the range of $900-1000 \mathrm{~km} \mathrm{~s}^{-1}$. If these velocities were due to the potential of NGC 3268 (and its associated dark matter), very radial orbits would be needed for surpassing the circular velocity that is about $300 \mathrm{~km} \mathrm{~s}^{-1}$ and probably constant (Caso et al. 2014). The high velocity offsets are, moreover, related to fairly large projected spatial offsets (which in the case of FS90224 is about $65 \mathrm{kpc}$ ), whereas the highest velocities are found at the bottom of the potential well. Furthermore, the galaxies do not show any morphological indication of being tidally disturbed. Moreover, there is no indication that the globular cluster system of NGC 3268 is tidally disturbed (Dirsch et al. 2003a), nor does the X-ray structure show any abnormality (Nakazawa et al. 2000). We therefore prefer the interpretation that the three S0s have to be placed in the background of NGC 3268. As Fig.7 suggests, more dwarf galaxies can be associated with this group, but some cases are ambiguous. There is the dwarf FS90-195 that hosts about ten point sources, which may be globular clusters. The radial velocity is $3495 \mathrm{~km} \mathrm{~s}^{-1}$, but it is located close to NGC 3268 (projected distance $15 \mathrm{kpc}$ ), so it may be bound and 
demonstrates how globular clusters can be donated to the system of NGC 3268. In that sense it could resemble SH2 in NGC 1316 (Richtler et al. 2012).

We note that NGC 3269 (FS90-184), together with NGC 3267 (FS90-168) and NGC 3271 (FS90-224), has been identified as the "Lyon Group of Galaxies (LGG)" 202 of Garcia (1993, 1995) and Barnes \& Webster (2001), who already suspected its location behind the Antlia cluster.

Galaxies of the low velocity group are mostly dwarf-like galaxies and might be bona fide in the foreground. All look quite discy, which may also indicate a less dense environment. The situation is somewhat different for the group around NGC 3258. There are also dwarfs (ANTLJ102914-353923.6 and FS90137 ) with positive velocity offsets of more than $1000 \mathrm{~km} \mathrm{~s}^{-1}$ to NGC 3258, but the brighter galaxies do not show such extreme radial velocities. There is also a group around FS90-226 that is less striking than the two dominant groups, which is also identified as a subgroup by Hess et al. (2015).

Therefore the likely explanation for Fig. 6 is that we are looking along a filament of galaxies and that at least a fraction of the galaxies in the three velocity groups apparent in Fig. 6 are separated by their recession velocities.

\subsection{NGC $3268 / 3258$ as fossil groups?}

If the crowding of galaxies around NGC 3268 is mainly a projection effect, this galaxy fulfils the criteria for being a fossil group. After applying the definition of Jones et al. (2003), it has the required X-ray luminosity of more than $10^{42} \mathrm{erg} \mathrm{s}^{-1}$, and the next galaxy in a luminosity ranking would be FS90-177, which is fainter than 2 mag in the $R$-band.

The brighter galaxies around NGC 3258 (FS90-105 and FS90-125) may be companions. FS90-125 is perhaps a magnitude fainter than NGC 3258, so only the X-ray criterium applies, and it cannot be called a fossil group. However, its globular cluster system is even richer than that of NGC 3268, so the idea that a galaxy group collapsed at very early times is plausible.

\subsection{Emission line galaxies}

Only three galaxies from our VIMOS sample present emission lines. One of them, FS90-131, was classified as a spiral. The other galaxies, FS90-220 and FS90-222, were classified as lenticulars. Evidence of strong star formation would not be expected if the environment of the Antlia gEs was dense: FS90131 is located at $5^{\prime}$ from NGC 3258, FS90-220 is $\sim 6^{\prime}$ from NGC 3268, and FS90-222 is $\sim 3^{\prime}$ from NGC 3273. Moreover, the $V_{\mathrm{R}, \mathrm{h}}$ in the three cases is lower than $1200 \mathrm{~km} \mathrm{~s}^{-1}$, which disqualifies them from dynamically belonging to these groups. Their velocities are better explained by recession velocities.

\section{Summary and conclusions}

We have presented new radial velocities, measured with VLTVIMOS and Gemini GMOS-S, for galaxies in the region normally addressed as the Antlia cluster. The fields are located in the surroundings of NGC 3258 and NGC 3268, the dominant galaxies of the cluster. Together with the literature data, our list of Antlia galaxies with measured radial velocities now embraces 105 galaxies. Large numbers of these objects are projected onto either of the two subgroups around NGC 3258 or NGC 3268. Because the gravitational potentials of these galaxies are constrained by X-ray studies and stellar dynamical tracers, we could compare the observed radial velocities with those expected. It turned out that the total range of velocities seems to be too high to be generated by the gravitational potential of NGC 3258 or NGC 3268. There are three groups of galaxies (not always clearly separated) characterised by radial velocities of about $1800 \mathrm{~km} \mathrm{~s}^{-1}, 2700 \mathrm{~km} \mathrm{~s}^{-1}$, and $3700 \mathrm{~km} \mathrm{~s}^{-1}$. We interpreted these velocities as recession velocities, which places the bright S0s around NGC 3268, in particular, into the background. Therefore, NGC 3268 might qualify as a fossil group, resembling, for example, NGC 4636 in the Virgo cluster in its properties. The intermediate velocity group (i.e. $V_{\mathrm{R}, \mathrm{h}}$ around $2700 \mathrm{~km} \mathrm{~s}^{-1}$ ) is the most populated one. The distances between NGC 3258 and NGC 3268 are not well constrained in the literature, and we cannot discard that the groups dominated by them are in the early stages of a merging process.

What we originally called the Antlia cluster is therefore characterised by several groups that, at least in some cases, are not gravitationally bound. We may be looking along a filament of the cosmic web.

Acknowledgements. We thank Ylva Schuberth for discussions about the radial velocities of Virgo galaxies, Mike Fellhauer for permission to use his orbit program, Francisco Azpillicueta for discussions of statistical issues, and Lilia Bassino for discussions about the Antlia cluster. We thank the refereee for suggestions that improved this article. This work was based on observations made with ESO telescopes at the La Silla Paranal Observatory under programmes ID 60.A-905 and ID 079.B-0480, and on observations obtained at the Gemini Observatory, which is operated by the Association of Universities for Research in Astronomy, Inc., under a cooperative agreement with the NSF on behalf of the Gemini partnership: the National Science Foundation (United States), the National Research Council (Canada), CONICYT (Chile), the Australian Research Council (Australia), Ministério da Ciência, Tecnologia e Inovação (Brazil) and Ministerio de Ciencia, Tecnología e Innovación Productiva (Argentina), and on observations acquired through the Gemini Science Archive. This research has made use of the NASA/IPAC Extragalactic Database (NED), which is operated by the Jet Propulsion Laboratory, California Institute of Technology, under contract with the National Aeronautics and Space Administration. This work was funded with grants from Consejo Nacional de Investigaciones Científicas y Técnicas de la República Argentina, Agencia Nacional de Promoción Científica y Tecnológica (BID AR PICT-2013-0317), and Universidad Nacional de La Plata (Argentina). T.R. is grateful for financial support from FONDECYT project Nr. 1100620, and from the BASAL Centro de Astrofísica y Tecnologías Afines (CATA) PFB-06/2007.

\section{References}

Agnello, A., Evans, N. W., \& Romanowsky, A. J. 2014, MNRAS, 442, 3284 Ashman, K. M., Bird, C. M., \& Zepf, S. E. 1994, AJ, 108, 2348

Barnes, D. G., \& Webster, R. L. 2001, MNRAS, 324, 859

Bassino, L. P., Richtler, T., \& Dirsch, B. 2008, MNRAS, 386, 1145

Blakeslee, J. P., Lucey, J. R., Barris, B. J., Hudson, M. J., \& Tonry, J. L. 2001, MNRAS, 327, 1004

Boschin, W., Girardi, M., Spolaor, M., \& Barrena, R. 2006, A\&A, 449, 461

Boschin, W., Barrena, R., \& Girardi, M. 2010, A\&A, 521, A78

Burgett, W. S., Vick, M. M., Davis, D. S., et al. 2004, MNRAS, 352, 605

Calderón, J. P., Bassino, L. P., Cellone, S. A., et al. 2015, MNRAS, 451, 791

Canterna, R. 1976, AJ, 81, 228

Cantiello, M., Blakeslee, J. P., Raimondo, G., et al. 2005, ApJ, 634, 239

Caso, J. P., Bassino, L. P., Richtler, T., Smith Castelli, A. V., \& Faifer, F. R. 2013, MNRAS, 430, 1088

Caso, J. P., Bassino, L. P., Richtler, T., Calderón, J. P., \& Smith Castelli, A. V. 2014, MNRAS, 442, 891

Colless, M., \& Dunn, A. M. 1996, ApJ, 458, 435

Conselice, C. J., Gallagher, III, J. S., \& Wyse, R. F. G. 2001, ApJ, 559, 791

Courteau, S., Cappellari, M., de Jong, R. S., et al. 2014, Rev. Mod. Phys., 86, 47

de Vaucouleurs, G., de Vaucouleurs, A., Corwin, Jr., H. G., et al. 1991, Third Reference Catalogue of Bright Galaxies. Volume I: Explanations and references. Volume II: Data for galaxies between $0^{\mathrm{h}}$ and $12^{\mathrm{h}}$, Volume III: Data for galaxies between $12^{\mathrm{h}}$ and $24^{\mathrm{h}}$

Dekel, A., Stoehr, F., Mamon, G. A., et al. 2005, Nature, 437, 707

Dirsch, B., Richtler, T., \& Bassino, L. P. 2003a, A\&A, 408, 929

Dirsch, B., Richtler, T., Geisler, D., et al. 2003b, AJ, 125, 1908

Dressler, A., \& Shectman, S. A. 1988, AJ, 95, 985 
Drinkwater, M. J., Gregg, M. D., \& Colless, M. 2001, ApJ, 548, L139 Edwards, S. A., Colless, M., Bridges, T. J., et al. 2002, ApJ, 567, 178 Evans, N. W., Wilkinson, M. I., Perrett, K. M., \& Bridges, T. J. 2003, ApJ, 583, 752

Ferguson, H. C., \& Sandage, A. 1990, AJ, 100, 1

Garcia, A. M. 1993, A\&AS, 100, 47

Garcia, A. M. 1995, A\&A, 297, 56

Grego, L., Carlstrom, J. E., Reese, E. D., et al. 2001, ApJ, 552, 2

Hess, K. M., Jarrett, T. H., Carignan, C., Passmoor, S. S., \& Goedhart, S. 2015, MNRAS, 452, 1617

Hopp, U., \& Materne, J. 1985, A\&AS, 61, 93

Hou, A., Parker, L. C., Wilman, D. J., et al. 2012, MNRAS, 421, 3594

Huchra, J. P., Macri, L. M., Masters, K. L., et al. 2012, ApJS, 199, 26

Jones, L. R., Ponman, T. J., Horton, A., et al. 2003, MNRAS, 343, 627

Jones, D. H., Read, M. A., Saunders, W., et al. 2009, MNRAS, 399, 683

Kim, S., Rey, S.-C., Jerjen, H., et al. 2014, ApJS, 215, 22

Kolmogorov, A. 1933, Giornale dell' 'Istituto Italiano degli Attuari, 4, 83

Lauberts, A., \& Valentijn, E. A. 1989, The surface photometry catalogue of the ESO-Uppsala galaxies

Longhetti, M., Rampazzo, R., Bressan, A., \& Chiosi, C. 1998, A\&AS, 130, 267

Mamon, G. A., \& Łokas, E. L. 2005a, MNRAS, 362, 95

Mamon, G. A., \& Łokas, E. L. 2005b, MNRAS, 363, 705
Mathewson, D. S., Ford, V. L., \& Buchhorn, M. 1992, ApJS, 81, 413

Matthews, L. D., Gallagher, III, J. S., \& Littleton, J. E. 1995, AJ, 110, 581

Muratov, A. L., \& Gnedin, O. Y. 2010, ApJ, 718, 1266

Nakazawa, K., Makishima, K., Fukazawa, Y., \& Tamura, T. 2000, PASJ, 52, 623

Ogando, R. L. C., Maia, M. A. G., Pellegrini, P. S., \& da Costa, L. N. 2008, AJ, 135,2424

Owers, M. S., Couch, W. J., \& Nulsen, P. E. J. 2009, ApJ, 693, 901

Pedersen, K., Yoshii, Y., \& Sommer-Larsen, J. 1997, ApJ, 485, L17

Pinkney, J., Roettiger, K., Burns, J. O., \& Bird, C. M. 1996, ApJS, 104, 1

Praton, E. A., \& Schneider, S. E. 1994, ApJ, 422, 46

Richtler, T., Kumar, B., Bassino, L. P., Dirsch, B., \& Romanowsky, A. J. 2012, A\&A, 543, L7

Royston, P. 1995, j-APPL-STAT, 44, 547

Sánchez-Blázquez, P., Peletier, R. F., Jiménez-Vicente, J., et al. 2006, MNRAS, 371, 703

Shapiro, S. S., \& Wilk, M. B. 1965, Biometrika, 52, 591

Smirnov, N. 1948, Ann. Math. Stat., 19, 279

Smith Castelli, A. V., Bassino, L. P., Richtler, T., et al. 2008, MNRAS, 386, 2311 Smith Castelli, A. V., Cellone, S. A., Faifer, F. R., et al. 2012, MNRAS, 419, 2472

Theureau, G., Bottinelli, L., Coudreau-Durand, N., et al. 1998, A\&AS, 130, 333 Xue, Y.-J., \& Wu, X.-P. 2000, ApJ, 538, 65 


\section{Appendix A: Additional tables}

Table A.1. Heliocentric radial velocities for background galaxies measured in this paper.

\begin{tabular}{lccc}
\hline \hline ID & $\begin{array}{c}\text { RA(J2000) } \\
\text { hh } \mathrm{mm} \mathrm{ss}\end{array}$ & $\begin{array}{c}\text { Dec(J2000) } \\
\mathrm{dd} \mathrm{mm} \mathrm{ss}\end{array}$ & $\begin{array}{c}V_{\mathrm{R}, \mathrm{h}} \\
\mathrm{km} \mathrm{s}^{-1}\end{array}$ \\
\hline FS90-75 & 102812.0 & -353220.4 & $12333 \pm 07$ \\
FS90-205 & 103018.5 & -352443.2 & $45907 \pm 30$ \\
ANTL102823-352754 & 102823.8 & -352754.0 & $40289 \pm 25$ \\
ANTL10294-352320 & 102904.6 & -352320.4 & $51147 \pm 18$ \\
ANTL10295-352146 & 102905.5 & -352146.8 & $52928 \pm 23$ \\
ANTL102817-353552 & 102817.0 & -353552.8 & $44240 \pm 85$ \\
ANTL102937-353552 & 102937.2 & -353552.8 & $7682 \pm 55$ \\
ANTL102936-353336 & 102936.7 & -353336.0 & $24420 \pm 50$ \\
ANTL102951-351210 & 102951.6 & -351210.8 & $32797 \pm 24$ \\
ANTL10303-3571 & 103003.8 & -35701.2 & $28192 \pm 21$ \\
ANTL103042-35914 & 103042.2 & -35914.4 & $15875 \pm 14$ \\
ANTL103049-352031 & 103049.4 & -352031.2 & $32830 \pm 23$ \\
ANTL103045-35161 & 103045.8 & -351601.2 & $32920 \pm 21$ \\
ANTL102945-35374 & 102945.4 & -353704.8 & $8900 \pm 19$ \\
ANTL102936-353336 & 102936.7 & -353336.0 & $24337 \pm 33$ \\
ANTL102926-351051 & 102926.9 & -351051.6 & $20041 \pm 10$ \\
ANTL103041-351250 & 103041.0 & -351250.4 & $9833 \pm 65$ \\
ANTL102940-35258 & 102940.6 & -352508.4 & $53700 \pm 42$ \\
ANTL102936-35266 & 102936.0 & -352606.0 & $24243 \pm 10$ \\
FS90-83 & 102823.0 & -353057.6 & $19670 \pm 18$ \\
FS90-88 & 102828.1 & -353104.8 & $19624 \pm 74$ \\
ANTL102838-352027 & 102838.2 & -352027.6 & $46732 \pm 54$ \\
\hline
\end{tabular}

Table A.2. Heliocentric radial velocity for Antlia members up to date.

\begin{tabular}{|c|c|c|c|c|c|}
\hline ID & NGC & $\begin{array}{c}\mathrm{RA}(\mathrm{J} 2000) \\
\text { hh mm ss }\end{array}$ & $\begin{array}{c}\operatorname{Dec}(\mathrm{J} 2000) \\
\mathrm{dd} \mathrm{mm} \mathrm{ss}\end{array}$ & Hubble type & $\begin{array}{c}V_{\mathrm{R}, \mathrm{h}} \\
\mathrm{km} \mathrm{s}^{-1} \\
\end{array}$ \\
\hline FS90-01 & & 102505.04 & -355858.8 & $\mathrm{SmV}$ & $3220 \pm 05^{1}$ \\
\hline FS90-18 & & 102644.88 & -365150.4 & SmIV & $2331 \pm 05^{1}$ \\
\hline FS90-28 & & 102702.16 & -345750.4 & SbII & $3402 \pm 08^{2}$ \\
\hline FS90-29 & & 102702.40 & -361330.0 & $\mathrm{Sc}$ & $3122 \pm 06^{2}$ \\
\hline FS90-44 & & 102721.12 & -351630.0 & So & $2931 \pm 45^{3}$ \\
\hline FS90-50 & & 102732.64 & -355909.6 & So & $2722 \pm 31^{3}$ \\
\hline FS90-64 & & 102757.84 & -354919.2 & dSB0 & $2261 \pm 45^{3}$ \\
\hline FS90-68 & & 102803.12 & -352631.2 & SBab & $\begin{array}{l}3219 \pm 18^{a} \\
3188 \pm 45^{3}\end{array}$ \\
\hline FS90-70 & & 102806.96 & -353520.4 & $\mathrm{dE}$ & $2864 \pm 70^{b}$ \\
\hline FS90-72 & & 102807.92 & -353820.4 & S0 & $2986 \pm 38^{b}$ \\
\hline FS90-77 & & 102815.12 & -353202.4 & $\mathrm{dE}, \mathrm{N}$ & $\begin{array}{l}2396 \pm 17^{a} \\
2382 \pm 49^{b}\end{array}$ \\
\hline FS90-79 & & 102819.2 & -352721.6 & S0 & $\begin{array}{l}2772 \pm 15^{a} \\
2930 \pm 60^{b}\end{array}$ \\
\hline FS90-80 & & 102818.96 & -354528.8 & dS0 & $2519 \pm 31^{3}$ \\
\hline ANTLJ102820-354236 & & 102819.68 & -354236.0 & $\mathrm{dE}, \mathrm{N}$ & $2904 \pm 40^{a}$ \\
\hline FS90-84 & & 102824.00 & -353140.8 & $\mathrm{E}$ & $\begin{array}{l}2489 \pm 26^{a} \\
2428 \pm 30^{4}\end{array}$ \\
\hline FS90-85 & & 102824.0 & -353422.8 & $\mathrm{dE}$ & $2000 \pm 200^{b}$ \\
\hline FS90-87 & & 102825.2 & -351434.8 & dE,N & $3429 \pm 13^{a}$ \\
\hline ANTLJ102829-351510.8 & & 102829.3 & -351510.8 & $\mathrm{dE}, \mathrm{N}$ & $3226 \pm 40^{a}$ \\
\hline FS90-93 & & 102831.92 & -354040.8 & $\mathrm{SmV}$ & $3608 \pm 57^{a}$ \\
\hline FS90-94 & & 102831.92 & -354221.6 & S0 & $\begin{array}{l}2791 \pm 24^{a} \\
2786 \pm 45^{3}\end{array}$ \\
\hline FS90-98 & & 102835.04 & -352739.6 & $\mathrm{BCD}$ & $2890 \pm 94^{b}$ \\
\hline FS90-103 & & 102845.12 & -353440.8 & $\mathrm{dE}$ & $2054 \pm 29^{b}$ \\
\hline
\end{tabular}

Notes. The upper index in Col. 6 indicates the reference for the $V_{\mathrm{R}, \mathrm{h}}$ measurement:

References. ${ }^{(a)}$ this paper; ${ }^{(b)}$ Smith Castelli et al. $(2008,2012)$. The rest of the measurements were obtained from NED, and their references are: (1) Matthews et al. (1995); (2) Theureau et al. (1998); ${ }^{(3)}$ Jones et al. (2009); (4) Huchra et al. (2012); ${ }^{(5)}$ de Vaucouleurs et al. (1991); ${ }^{(6)}$ Ogando et al. (2008); ${ }^{(7)}$ Mathewson et al. (1992); ${ }^{(8)}$ Lauberts \& Valentijn (1989); ${ }^{(9)}$ Longhetti et al. (1998). 
Table A.2. continued.

\begin{tabular}{|c|c|c|c|c|c|}
\hline ID & NGC & $\begin{array}{l}\text { RA(J2000) } \\
\text { hh mm ss }\end{array}$ & $\begin{array}{c}\operatorname{Dec}(\mathrm{J} 2000) \\
\mathrm{dd} \mathrm{mm} \mathrm{ss}\end{array}$ & Hubble type & $\begin{array}{c}V_{\mathrm{R}, \mathrm{h}} \\
\mathrm{km} \mathrm{s}^{-1}\end{array}$ \\
\hline FS90-105 & 3257 & 102848.0 & -353928.8 & SB01 & $\begin{array}{l}3237 \pm 15^{a} \\
3200 \pm 26^{b}\end{array}$ \\
\hline FS90-106 & & 102851.36 & -350939.6 & BCD & $2409 \pm 115^{b}$ \\
\hline FS90-108 & & 102853.28 & -351912.0 & $\mathrm{dE}, \mathrm{N}$ & $2611 \pm 39^{b}$ \\
\hline FS90-109 & & 102853.04 & -353252.8 & $\mathrm{dE}$ & $\begin{array}{l}1632 \pm 32^{a} \\
1618 \pm 24^{b}\end{array}$ \\
\hline FS90-110 & & 102853.04 & -353534.8 & M32 & $2911 \pm 7^{b}$ \\
\hline FS90-111 & 3258 & 102854.00 & -353621.6 & E & $\begin{array}{l}2792 \pm 50^{a} \\
2792 \pm 28^{b}\end{array}$ \\
\hline FS90-120 & & 102902.16 & -353404.8 & $\operatorname{ImV}$ & $\begin{array}{l}2721 \pm 30^{a} \\
2634 \pm 13^{b}\end{array}$ \\
\hline FS90-123 & & 102903.12 & -354030.0 & $\mathrm{dE}, \mathrm{N}$ & $1865 \pm 25^{b}$ \\
\hline FS90-125 & 3260 & 102906.24 & -353534.8 & S02 & $2439 \pm 46^{b}$ \\
\hline ANTLJ102910-353920.1 & & 102910.32 & -353921.6 & $\mathrm{dE}, \mathrm{N}$ & $1940 \pm 155^{b}$ \\
\hline FS90-131 & & 102911.04 & -354124.0 & $\mathrm{Sb}(\mathrm{r})$ & $\begin{array}{l}2158 \pm 10^{a} \\
2104 \pm 60^{b}\end{array}$ \\
\hline FS90-133 & & 102912.00 & -353928.8 & $\mathrm{dE}, \mathrm{N}$ & $\begin{array}{l}2219 \pm 15^{a} \\
2205 \pm 24^{b}\end{array}$ \\
\hline FS90-134 & & 102913.20 & -352924.0 & S0 & $1355 \pm 60^{b}$ \\
\hline ANTLJ102914-353923.6 & & 102914.40 & -353925.2 & $\mathrm{dE}, \mathrm{N}$ & $4067 \pm 115^{b}$ \\
\hline FS90-136 & & 102915.36 & -352558.8 & $\mathrm{dE}, \mathrm{N}$ & $\begin{array}{l}2995 \pm 16^{a} \\
2989 \pm 10^{b}\end{array}$ \\
\hline FS90-137 & & 102915.12 & -354134.8 & $\operatorname{ImV}$ & $3987 \pm 36^{b}$ \\
\hline FS90-139 & & 102915.60 & -350404.8 & $\mathrm{dE}, \mathrm{N}$ & $1950 \pm 55^{3}$ \\
\hline FS90-140 & & 102918.24 & -35356.0 & $\mathrm{dE}, \mathrm{N}$ & $1924 \pm 31^{b}$ \\
\hline FS90-142 & & 102920.16 & -353509.6 & dS0 & $2245 \pm 13^{b}$ \\
\hline FS90-152 & & 102928.86 & -344022.8 & $\mathrm{E}$ & $4093 \pm 45^{3}$ \\
\hline FS90-153 & & 102931.44 & -351539.6 & S0 & $\begin{array}{l}1785 \pm 13^{a} \\
1733 \pm 39^{b}\end{array}$ \\
\hline FS90-159 & & 102941.52 & -351731.2 & $\mathrm{dE}, \mathrm{N}$ & $2821 \pm 21^{a}$ \\
\hline FS90-162 & & 102943.44 & -352949.2 & $\mathrm{dE}, \mathrm{N}$ & $2933 \pm 31^{a}$ \\
\hline FS90-165 & & 102946.08 & -354225.2 & S0 & $\begin{array}{l}2695 \pm 14^{a} \\
2604 \pm 45^{3}\end{array}$ \\
\hline FS90-168 & 3267 & 102948.48 & -351922.8 & SB01/2 & $3709 \pm 33^{b}$ \\
\hline FS90-169 & & 102948.48 & -352512.0 & $\mathrm{E}$ & $2999 \pm 37^{b}$ \\
\hline FS90-172 & & 102951.84 & -345436.0 & S0 & $2549 \pm 19^{5}$ \\
\hline FS90-173 & & 102951.60 & -351004.8 & $\mathrm{dE}$ & $\begin{array}{l}2677 \pm 32^{a} \\
2609 \pm 45^{3}\end{array}$ \\
\hline FS90-175 & & 102953.52 & -352237.2 & dSB01 & $\begin{array}{l}1834 \pm 25^{a} \\
1781 \pm 66^{b}\end{array}$ \\
\hline FS90-176 & & 102954.48 & -351716.8 & $\mathrm{dE}, \mathrm{N}$ & $1751 \pm 34^{a}$ \\
\hline FS90-177 & & 102954.48 & -351919.2 & $\mathrm{dE}, \mathrm{N}$ & $\begin{array}{c}3540 \pm 9^{a} \\
3505 \pm 45^{b}\end{array}$ \\
\hline FS90-184 & 3269 & 102957.60 & -351330.0 & S0/a & $3754 \pm 33^{b}$ \\
\hline FS90-185 & 3268 & 102958.56 & -351930.0 & $\mathrm{E}$ & $2800 \pm 21^{b}$ \\
\hline FS90-186 & & 102959.52 & -351810.8 & $\mathrm{dE}$ & $3721 \pm 45^{a}$ \\
\hline FS90-187 & & 103001.20 & -354854.0 & dS0 & $1960 \pm 55^{3}$ \\
\hline FS90-188 & & 103002.40 & -352428.8 & $\mathrm{dE}$ & $2673 \pm 17^{b}$ \\
\hline FS90-192 & & 103004.56 & -352031.2 & M32 & $\begin{array}{c}2511 \pm 18^{a} \\
2526 \pm 4^{b}\end{array}$ \\
\hline FS90-195 & & 103006.48 & -351825.2 & $\mathrm{dE}$ & $3495 \pm 64^{a}$ \\
\hline FS90-196 & & 103006.48 & -352331.2 & $\mathrm{dE}$ & $3593 \pm 9^{b}$ \\
\hline ANTLJ103013-352458.3 & & 103013.92 & -352457.6 & $\mathrm{dE}, \mathrm{N}$ & $2613 \pm 200^{b}$ \\
\hline FS90-208 & & 103018.72 & -351149.2 & S0 & $1774 \pm 100^{b}$ \\
\hline FS90-209 & & 103019.44 & -353448.0 & $\mathrm{dE}$ & $3065 \pm 13^{b}$ \\
\hline FS90-212 & & 103021.36 & -353531.2 & SmIII & $2364 \pm 27^{b}$ \\
\hline ANTLJ103021-35314.8 & & 103021.38 & -353104.8 & $\mathrm{dE}, \mathrm{N}$ & $2707 \pm 19^{a}$ \\
\hline FS90-213 & & 103021.60 & -351214.4 & $\mathrm{dE}$ & $\begin{array}{l}2151 \pm 60^{a} \\
2185 \pm 21^{b}\end{array}$ \\
\hline ANTLJ103022-353806 & & 103022.08 & -353806.0 & $\mathrm{dE}, \mathrm{N}$ & $3405 \pm 40^{a}$ \\
\hline FS90-216 & & 103022.56 & -351026.4 & $\mathrm{E}$ & $\begin{array}{c}2957 \pm 22^{a} \\
2944 \pm 103^{b}\end{array}$ \\
\hline FS90-219 & & 103024.72 & -350632.4 & $\mathrm{Sb}$ & $1781 \pm 45^{3}$ \\
\hline
\end{tabular}


Table A.2. continued.

\begin{tabular}{|c|c|c|c|c|c|}
\hline ID & NGC & $\begin{array}{l}\text { RA(J2000) } \\
\text { hh mm ss }\end{array}$ & $\begin{array}{c}\operatorname{Dec}(\mathrm{J} 2000) \\
\mathrm{dd} \mathrm{mm} \mathrm{ss}\end{array}$ & Hubble type & $\begin{array}{r}V_{\mathrm{R}, \mathrm{h}} \\
\mathrm{km} \mathrm{s}^{-1} \\
\end{array}$ \\
\hline FS90-220 & & 103024.72 & -351518.0 & S0/a & $\begin{array}{c}1160 \pm 8^{a} \\
1182 \pm 45^{3}\end{array}$ \\
\hline FS90-222 & & 103025.44 & -353343.2 & S0/a & $\begin{array}{c}2077 \pm 6^{a} \\
2140 \pm 45^{3}\end{array}$ \\
\hline FS90-223 & & 103025.68 & -351319.2 & $\mathrm{dE}, \mathrm{N}$ & $\begin{array}{c}2701 \pm 29^{a} \\
2661 \pm 9^{b}\end{array}$ \\
\hline FS90-224 & 3271 & 103026.64 & -352136.0 & $\mathrm{Sb} 02$ & $3737 \pm 27^{b}$ \\
\hline FS90-226 & 3273 & 103029.28 & -353636.0 & S0/a & $\begin{array}{l}2660 \pm 08^{a} \\
2503 \pm 20^{6}\end{array}$ \\
\hline FS90-227 & & 103031.44 & -352306.0 & $\mathrm{dE}$ & $\begin{array}{l}2948 \pm 34^{a} \\
2921 \pm 60^{b}\end{array}$ \\
\hline FS90-228 & & 103031.68 & -351438.4 & dE,N & $\begin{array}{l}2450 \pm 18^{a} \\
2417 \pm 13^{b}\end{array}$ \\
\hline ANTLJ103033-352638.6 & & 103033.36 & -352638.4 & $\mathrm{dE}, \mathrm{N}$ & $2311 \pm 130^{b}$ \\
\hline FS90-231 & & 103034.56 & -352313.2 & $\mathrm{dE}, \mathrm{N}$ & $\begin{array}{l}2915 \pm 20^{a} \\
2909 \pm 38^{b}\end{array}$ \\
\hline ANTLJ103036-353046.8 & & 103036.48 & -353046.8 & $\mathrm{dE}, \mathrm{N}$ & $3394 \pm 27^{a}$ \\
\hline ANTLJ103037-352708.8 & & 103037.44 & -352707.2 & $\mathrm{dE}, \mathrm{N}$ & $2400 \pm 100^{b}$ \\
\hline FS90-238 & & 103045.6 & -352132.4 & $\mathrm{Sm}$ & $3078 \pm 37^{a}$ \\
\hline ANTLJ103047-353918 & & 103046.8 & -353918.0 & $\mathrm{dE}, \mathrm{N}$ & $3412 \pm 32^{a}$ \\
\hline FS90-241 & & 103048.48 & -353220.4 & $\mathrm{dE}, \mathrm{N}$ & $3518 \pm 36^{a}$ \\
\hline FS90-244 & & 103051.60 & -364413.2 & $\mathrm{SBb}(\mathrm{r}) \mathrm{II}$ & $3161 \pm 45^{3}$ \\
\hline FS90-253 & & 103100.24 & -343350.4 & SB0 & $2111 \pm 18^{5}$ \\
\hline FS90-258 & & 103103.12 & -344015.6 & $\mathrm{dE}, \mathrm{N}$ & $2450 \pm 55^{3}$ \\
\hline FS90-277 & & 103124.72 & -351315.6 & $\mathrm{SBb}(\mathrm{rs}) \mathrm{II}$ & $2597 \pm 19^{5}$ \\
\hline FS90-298 & & 103148.48 & -360144.4 & $\mathrm{Sd}$ & $3168 \pm 38^{4}$ \\
\hline FS90-300 & & 103152.08 & -345114.4 & $\mathrm{Sa}$ & $3200 \pm 22^{2}$ \\
\hline FS90-301 & & 103151.84 & -351214.4 & So & $2423 \pm 45^{3}$ \\
\hline FS90-304 & & 103155.92 & -352432.4 & $\mathrm{Sa}$ & $2476 \pm 16^{2}$ \\
\hline FS90-306 & & 103156.16 & -345927.6 & SB0 & $1981 \pm 45^{3}$ \\
\hline FS90-307 & & 103157.12 & -345345.6 & $\mathrm{dE}$ & $2784 \pm 30^{a}$ \\
\hline FS90-309 & & 103159.04 & -351145.6 & SB0 & $2289 \pm 19^{5}$ \\
\hline FS90-318 & & 103208.40 & -344015.6 & $\mathrm{dE}, \mathrm{N}$ & $2588 \pm 45^{3}$ \\
\hline FS90-321 & & $1032 \quad 12.24$ & -344008.4 & SB0 & $2129 \pm 45^{3}$ \\
\hline FS90-323 & & 103214.88 & -351528.8 & $\mathrm{Sm}$ & $3825 \pm 45^{3}$ \\
\hline FS90-325 & & 103225.20 & -350000.0 & $\mathrm{Sd}$ & $3058 \pm 10^{7}$ \\
\hline FS90-331 & & 103259.28 & -345258.8 & So & $2779 \pm 19^{5}$ \\
\hline FS90-341 & & 103401.20 & -351655.2 & Sa pec & $2573 \pm 08^{8}$ \\
\hline FS90-343 & & 103407.20 & -351926.4 & SO & $2754 \pm 09^{9}$ \\
\hline FS90-345 & & 103413.68 & -361355.2 & So & $3372 \pm 45^{3}$ \\
\hline FS90-353 & & 103455.44 & $\begin{array}{ll}-3528 & 19.2\end{array}$ & $\mathrm{Sd}$ & $2662 \pm 45^{3}$ \\
\hline FS90-373 & & 103722.80 & -352136.0 & $\mathrm{dE}, \mathrm{N}$ & $2349 \pm 55^{3}$ \\
\hline
\end{tabular}

